\title{
THE EFFECT OF TORSIONAL RIGIDITY BETWEEN ELEMENTS ON FREE VIBRATIONS OF A TELESCOPIC HYDRAULIC CYLINDER SUBJECTED TO EULER'S LOAD
}

\author{
Sebastian Uzny ${ }^{1}$, Lukasz Kutrowski ${ }^{2}$ \\ Institute of Mechanics and Machine Design Fundamentals, Czestochowa University of Technology \\ Czestochowa, Poland \\ uzny@imipkm.pcz.pl,kutrowski@imipkm.pcz.pl
}

Received: 29 June 2017; Accepted: 11 September 2017

\begin{abstract}
The boundary problem of free vibrations of a hydraulic telescopic cylinder, subjected to Euler's load was presented in this work. The task was formulated on the basis of the Hamilton's principle. The computational model, formulated by Tomski, was taken into account during analysis. Numerical calculations concern free vibrations of multi-stage hydraulic telescopic cylinder. Results were presented in the form of characteristic curves on the plane: external load-natural vibration frequency. Non-dimensional parameters of the structure were defined in reference to the characteristic parameters of the piston rod. During numerical simulations, an influence of a non-dimensional stiffness parameter between following elements on free vibrations of a hydraulic telescopic cylinder was determined for a different number of cylinder stages.
\end{abstract}

MSC 2010: 74H45, $65 P 99$

Keywords: free vibrations, hydraulic telescopic cylinder, slender system

\section{Introduction}

Hydraulic cylinders are certain kinds of motors, which convert the energy of compressed hydraulic fluid to mechanical energy. A typical example is a linear single-acting cylinder, which is under consideration in this thesis. One-stage and multi-stage (telescopic) cylinders can be found depending on the number of stages. Telescopic cylinders are used when a long output range and a very compact retracted length are required. They are used especially in dump trucks (dump body) and hydraulic elevators.

Two different computational models concerning the stability and vibrations of hydraulic cylinders were developed by Tomski $[1,2]$. The first model refers to free transversal vibrations and static stability of cylinders. It is used for cylinders with a high slenderness ratio [1]. The second model refers to free longitudinal vibrations 
and forced vibrations. This kind of model is used for a cylinder with a low slenderness ratio [2]. The limiting values of parameters, when the computational model has to be changed can be assumed when free transversal and longitudinal vibrations are equal. Free transversal vibrations of cylinders, formulated on the basis of the first computational model, were considered in works [1-6]. The results of numerical simulations according to the one-stage hydraulic cylinders for different boundary conditions and parameters of structure can be found in those papers. Parameters of cylinders are: degree of coverage, stiffness of fixed, stiffness of piston rod and cylinders, stiffness of sealing and guiding elements. In paper [3] longitudinal inertia of the piston rod has been taken into account due to analysis. The effect of pressure along the cylinder, which has significant impact on its strength was presented in work [4]. In papers [5, 6] the results of experimental analysis of one-stage hydraulic cylinder were carried out what confirmed the proposed computational model.

In this paper, the effect of rigidity between the following elements (sealing and guiding elements for piston rod and all of cylinders) on characteristic curves is analysed. In the numerical research the different number of stages (one, three, five stage hydraulic cylinders) were taken into consideration.

\section{Boundary value problem}

The considered n-stage telescopic hydraulic cylinder subjected to Euler's load is presented in Figure 1. Structure consists of $\mathrm{n}$ cylinders and the piston rod. The overall length of the configuration was defined as $l_{C}$.

In this work, the telescopic hydraulic cylinder is considered as fully extended and simply supported on both ends. The torsional rigidity of sealing and guiding elements was modelled by rotational springs of $C_{R i}$ stiffness. Stiffness of rotational springs are as follows: $C_{R 1}=C_{R 2}=C_{R 3}=C_{R 4}=C_{R 5}=C_{R}$. Geometrical dimensions of the cylinders (outer diameter $d_{z i}$ and inner diameter $d_{w i}$ ) were defined as:

$$
\begin{aligned}
& d_{z i}=d_{t}+2(n-i) g_{U}+2(n-i) g_{R} \\
& d_{w i}=d_{t}+2(n-i) g_{U}+2(n-i-1) g_{R}
\end{aligned}
$$

where $g_{U}$ and $g_{R}$ stand for the thickness of the sealing element and cylinder.

Each element of the hydraulic cylinder is characterized by adequate flexural rigidity $(E J)_{i}(E$ - Young's modulus, $J$ - geometrical inertia moment) and mass per length unit $(\rho A)_{i}(\rho$ - density, $A$ - area of cross section). The mass of the hydraulic fluid, which fills the cylinders is $(\rho A)_{c i}\left((\rho A)_{c n}=0\right)$, masses of sealing and guiding elements $m_{i}$ were taken into consideration. Elements of the structure marked as $i=1,2, \ldots, n-1$ correspond to cylinders and the $n$-element correspond to the piston rod. 
The boundary problem has been formulated on the basis of Hamilton's principle.

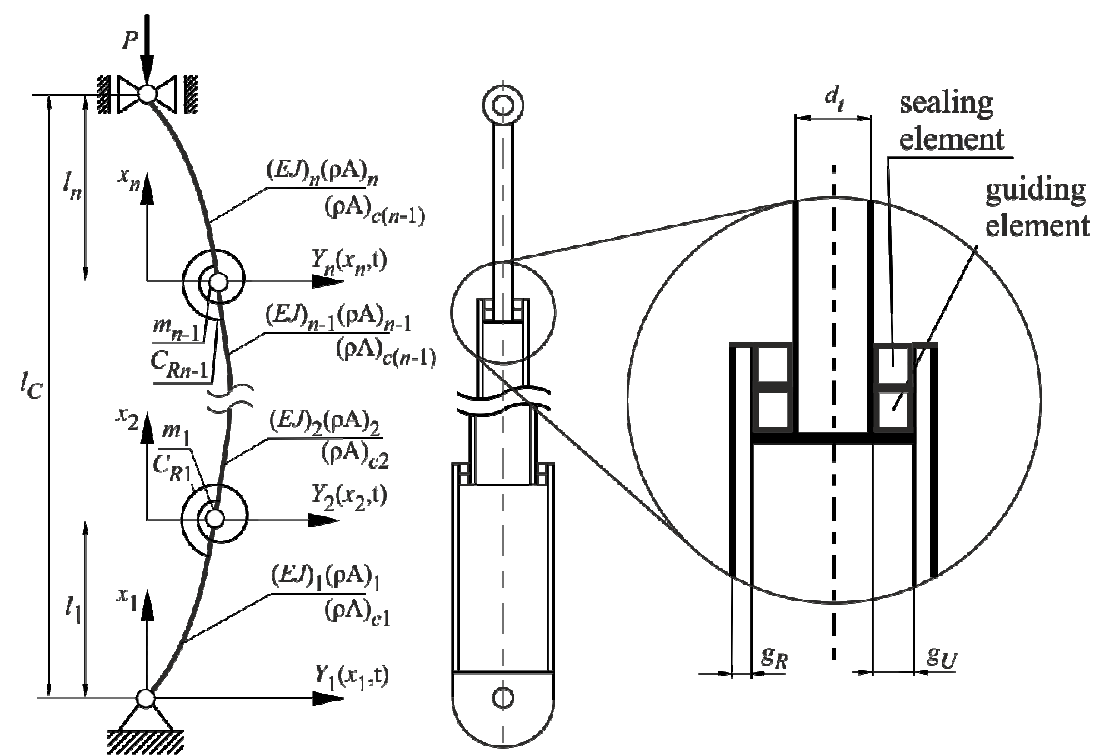

Fig. 1. Scheme of n-stage telescopic hydraulic cylinder subjected to Euler's load

Potential energy $(V)$ and kinetic energy $(T)$ are as follows:

$$
\begin{aligned}
& V=\frac{1}{2} \sum_{i=1}^{n} \int_{0}^{l_{i}}(E I)_{i}\left(\frac{\partial^{2} Y_{i}\left(x_{i}, t\right)}{\partial x_{i}^{2}}\right)^{2} d x_{i}-\frac{1}{2} P \sum_{i=1}^{n} \int_{0}^{l_{i}}\left(\frac{\partial Y_{i}\left(x_{i}, t\right)}{\partial x_{i}}\right)^{2} d x_{i}+ \\
& +\frac{1}{2} C_{R i} \sum_{i=1}^{n-1}\left[\left.\frac{\partial Y_{i}\left(x_{i}, t\right)}{\partial x_{i}}\right|^{x_{i}=l_{i}}-\left.\frac{\partial Y_{i+1}\left(x_{i+1}, t\right)}{\partial x_{i+1}}\right|_{x_{i+1}=0}\right]^{2} \\
& T=\frac{1}{2} \sum_{i=1}^{n}(\rho A)_{i} \int_{0}^{l_{i}}\left(\frac{\partial Y_{i}\left(x_{i}, t\right)}{\partial t}\right)^{2} d x_{i}+\frac{1}{2} \sum_{i=1}^{n-1} m_{i}\left(\left.\frac{\partial Y_{i}\left(x_{i}, t\right)}{\partial t}\right|^{x=l_{i}}\right)^{2}+ \\
& \frac{1}{2} \sum_{i=1}^{n}(\rho A)_{c i} \int_{0}^{l_{i}}\left(\frac{\partial Y_{i}\left(x_{i}, t\right)}{\partial t}\right)^{2} d x_{i}
\end{aligned}
$$

Taking into account the potential and kinetic energy in Hamilton principle, after appropriate transformations, differential equations of motion and natural boundary conditions are obtained. Differential equations of motion after separation of variables can be written as follows: 


$$
\begin{aligned}
& (E I)_{i} \frac{d^{4} y_{i}\left(x_{i}\right)}{d x_{i}^{4}}+P \frac{d^{2} y_{i}\left(x_{i}\right)}{d x_{i}^{2}}-(\rho A)_{i} \omega^{2} \frac{d^{2} y_{i}\left(x_{i}\right)}{d x_{i}^{2}}+ \\
& -(\rho A)_{c i} \omega^{2} \frac{d^{2} y_{i}\left(x_{i}\right)}{d x_{i}^{2}}=0 \quad \text { for } i=1 \ldots n
\end{aligned}
$$

where $\omega$ is free vibrations frequency.

Boundary conditions (geometrical and natural) are expressed as follows:

$$
\begin{gathered}
y_{1}(0)=0 ; y_{n}\left(l_{n}\right)=0 ; y_{i}\left(l_{i}\right)=y_{i+1}\left(l_{i+1}\right) \\
\left.(E I)_{1} \frac{d^{2} y_{1}\left(x_{1}\right)}{d^{2} x_{1}}\right|^{x_{1}=0}=0 ;-\left.(E I)_{n} \frac{d^{2} y_{n}\left(x_{n}\right)}{d^{2} x_{n}}\right|^{x_{n}=l_{n}}=0 \\
-\left.(E I)_{i} \frac{d^{2} y_{i}\left(x_{i}\right)}{d x_{i}^{2}}\right|^{x_{i}=l_{i}}-C_{R i}\left[\left.\frac{d y_{i}\left(x_{i}\right)}{d x_{i}}\right|^{x_{i}=l_{i}}-\left.\frac{d y_{i+1}\left(x_{i+1}\right)}{d x_{i+1}}\right|_{x_{i+1}=0}\right]=0 \\
\left.(E I)_{i+1} \frac{d^{2} y_{i+1}\left(x_{i+1}\right)}{d x_{i+1}^{2}}\right|_{x_{i+1}=0}+C_{R i}\left[\left.\frac{d y_{i}\left(x_{i}\right)}{d x_{i}}\right|^{x_{i}=l_{i}}-\left.\frac{d y_{i+1}\left(x_{i+1}\right)}{d x_{i+1}}\right|_{x_{i+1}=0}\right]=0 \\
\left.(E I)_{i} \frac{d^{3} y_{i}\left(x_{i}\right)}{d x_{i}^{3}}\right|^{x_{i}=l_{i}}-\left.(E I)_{i+1} \frac{d^{3} y_{i+1}\left(x_{i+1}\right)}{d x_{i+1}^{3}}\right|_{x_{i+1}=0}+\left.P \frac{d y_{i}\left(x_{i}\right)}{d x_{i}}\right|^{x_{i}=l_{i}}+ \\
-\left.P \frac{d y_{i+1}\left(x_{i+1}\right)}{d x_{i+1}}\right|_{x_{i+1}=0}+\left.m_{i} \omega^{2} y_{i}\left(x_{i}\right)\right|^{x_{i}=l_{i}}=0
\end{gathered}
$$

Solutions of differential equations (4) can be written as:

$$
y_{i}\left(x_{i}\right)=A_{i} \cosh \left(\alpha_{i} x_{i}\right)+B_{i} \sinh \left(\alpha_{i} x_{i}\right)+C_{i} \cos \left(\beta_{i} x_{i}\right)+D_{i} \sin \left(\beta_{i} x_{i}\right)
$$

where:

$$
\begin{gathered}
\alpha_{i}=\sqrt{-\frac{k_{i}^{2}}{2}+\sqrt{\frac{k_{i}^{4}}{4}+\Omega_{i}^{2}}} ; \beta_{i}=\sqrt{\frac{k_{i}^{2}}{2}+\sqrt{\frac{k_{i}^{4}}{4}+\Omega_{i}^{2}}} \\
k_{i}{ }^{2}=\frac{P}{(E I)_{i}} \quad ; \quad \Omega_{i}{ }^{2}=\frac{(\rho A)_{i} \omega^{2}}{(E I)_{i}}
\end{gathered}
$$

After substitution of the solution (6) into boundary conditions, the system of equations is obtained. The matrix determinant of coefficients equated to zero leads to the transcendental equation, from which the natural vibration frequency of the system can be determined. 


\section{Results}

Results of numerical simulations of free vibrations of the considered telescopic hydraulic cylinder were presented in the non-dimensional form, defined as:

$$
\zeta_{G U}=\frac{g_{U}}{d_{t}} ; \zeta_{G R}=\frac{g_{R}}{d_{t}} ; c=\frac{C_{R} l_{C}}{(E I)_{n}} ; \lambda=\frac{P l_{C}{ }^{2}}{(E I)_{n}} ; \Omega^{*}=\frac{\omega^{2}(\rho A)_{n} l_{C}{ }^{4}}{(E I)_{n}}
$$

a) $\lambda$

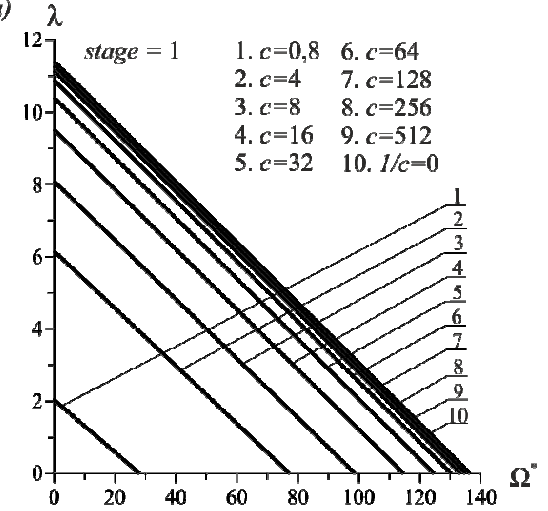

b) $\lambda$

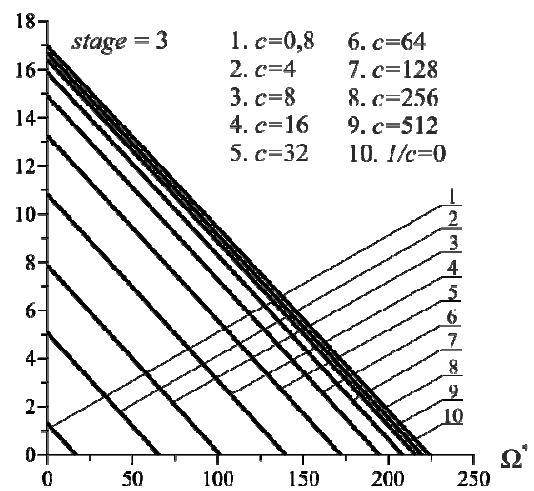

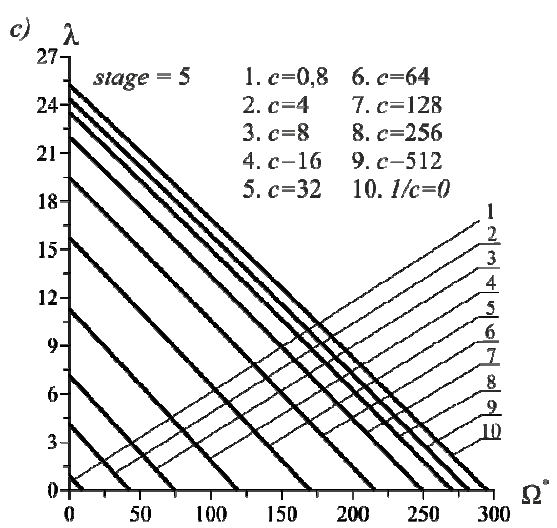

Fig. 2a-c. Characteristic curves on non-dimensional plane for different parameters of stiffness between elements $\left(d_{t}=0.1 \mathrm{~m}, \zeta_{G U}=0.05, \zeta_{G R}=0.1\right)$

In Figure 2 characteristic curves $\left(\lambda_{c r}\left(\Omega^{*}\right)\right)$ in case of: one-stage cylinder (Fig. 2a), three-stage cylinder (Fig. 2b), five-stage cylinder (Fig. 2c) are presented. Numerical calculations were carried out for chosen cylinder parameters $\left(d_{t}=0.1 \mathrm{~m}\right.$, $\left.\zeta_{G U}=0.05, \zeta_{G R}=0.1\right)$ and for different values of a non-dimensional parameter of rotational spring stiffness $c(c=0.8 ; 4 ; 8 ; 16 ; 32 ; 64 ; 128 ; 256 ; 518 ; 1 / c=0)$. An influence of the considered stiffness of rotational nodes in which the ends of individual hydraulic cylinder elements are joined, on natural vibration frequency magnitude depends on this stiffness as well as on the number of stages. 


\section{Conclusions}

The boundary problem of free vibrations of a telescopic hydraulic cylinder subjected to Euler's load was considered in this paper. The results were plotted in the form of characteristic curves. An influence of the guiding and sealing elements stiffness on dynamic behaviour were analysed.

On the basis of the obtained results, it can be concluded that the stiffness of the sealing and guiding elements have great influence on vibration frequency and critical load (in the presented problem the critical load corresponds to the zero magnitude of the vibration frequency - kinetic stability criterion). The smaller the rotational node stiffness the greater its influence on vibration frequency and critical load. On the basis of the proposed non-dimensional parameters, the obtained relation load - vibration frequency is linear. The characteristic curves are parallel to each other at the considered configuration of the system. Reduction of stiffness of guiding and sealing elements due to wear or damage can have serious consequences in further exploitation of the hydraulic cylinders because the reduction of loading capacity as well as vibration frequency can be observed.

\section{Acknowledgement}

The study has been carried out within the statutory funds of the Czestochowa University of Technology (BS/PB-1-101-3020/11/P).

\section{References}

[1] Tomski L., Elastic carrying capacity of a hydraulic prop, Engineering Transactions 1977, 25(2), 247-263.

[2] Tomski L., Dynamika stojaków hydraulicznych obudów górniczych, Praca habilitacyjna, Nr 17, Częstochowa 1979.

[3] Sochacki W., Tomski L., Free vibration and dynamic stability of a hydraulic cylinder set, Machine Dynamics Problems 1999, 23(4), 91-104.

[4] Tomski L., Elastic stability of hydraulic props of longwall supports, Archives of Minning Science 1978, 23(3), 217-231.

[5] Tomski L., Uzny S., A Hydraulic cylinder subjected to euler's load in aspect of the stability and free vibrations taking into account discrete elastic elements, Archives of Civil and Mechanical Engineering 2011, 11, 3, pp. 769-785.

[6] Uzny S., Free vibrations and stability of hydraulic cylinder fixed elastically on both ends, Proc. Appl. Math. Mech. 2009, 9, 303-304. 